\title{
Curvature of Determinant Bundles for Degenerate Families
}

\author{
Yue Lin L. Tong ${ }^{1, ~}$, I-Hsun Tsai ${ }^{2, \star \star}$ \\ ${ }^{1}$ Purdue University, West Lafayette, IN 47907, USA \\ ${ }^{2}$ National Taiwan University, Taipei, Taiwan, Republic of China
}

Received: 7 February 1994/in revised form: 24 August 1994

\begin{abstract}
We calculate the $(1,1)$ curvature of the Beilinson Schechtman connection for the determinant bundle associated to a family of Riemann surfaces with ordinary singularities. As consequences we obtain generalizations of theorems of Bismut and Bost.
\end{abstract}

\section{Introduction}

In this paper we calculate the $(1,1)$ curvature of the Beilinson Schechtman connection $\nabla_{B S}$ [BS] for the determinant bundle $\lambda_{E}$ associated to a vector bundle $E$ over a family $\pi: X \longrightarrow S$ of Riemann surfaces with ordinary singularities. Given smooth connections $\nabla_{E}$ on $E$ and $\nabla_{\omega}$ on $\omega_{X / S}$ which then induces a connection $\nabla_{\omega^{-1}}$ on $T Z$ (tangents to fiber outside singular set), we show that the connection form of $\nabla_{B S}$ is $L^{1}$ on $S$ and its distributional derivative is

$$
\frac{i}{2 \pi} \overline{\partial_{S}} \nabla_{B S}=\int_{X / S}\left[\operatorname{Td}\left(T Z, \nabla_{\omega^{-1}}\right) \operatorname{ch}\left(E, \nabla_{E}\right)\right]_{2}+\frac{r k(E)}{12} \delta_{\pi(\Sigma)},
$$

where []$_{2}$ denotes component of bidegree $(2,2)$ and $\delta_{\pi(\Sigma)}$ is the delta function of the singular locus (with multiplicities).

In case $\nabla_{\omega}$ and $\nabla_{E}$ arise from hermitian metrics, we showed in [TTs] that $\nabla_{B S}$ specializes to $\nabla_{Q}$ the Quillen connection. Thus as a consequence we obtain a generalization of the main theorem of Bismut Bost [BB, Theorem 2.1], where they established (1) for $\nabla_{Q}$.

The proof in $[\mathrm{BB}]$ relies on global methods to estimate analytic torsion and first nonzero eigenvalue of the Laplacian. Furthermore it requires a reduction to the projective case. By contrast $\nabla_{B S}$ is expressed in terms of fiber integrals of parametrices which have local differential geometric formulas in terms of $\nabla_{\omega^{-1}}, \nabla_{E}$ and liftings of vector fields from $S$ to $X$. It turns out that upon expanding these local formulas near singular points one finds rather directly (cf. Lemma 2.3) the terms

$\star$ Supported in part by NSF Grant No DMS-9201022.

$\star \star$ Supported in part by NSC Grant No 83-0208-M-002-039, Republic of China. 
with highest order poles which give rise to the correction $\frac{r k(E)}{12} \delta_{\pi(\Sigma)}$. Thus it seems that the Beilinson Schechtman connection is particularly suited to dealing with the singular case. To justify the calculations we need to incorporate the following. First of all, in Sect. 1, we extend the Beilinson Schechtman trace complex to families with ordinary double points. Using local coordinates fitted to ordinary singularities we find a natural complex whose cohomology gives the Atiyah algebra $\mathscr{A}_{\lambda_{E}}$ with logarithmic singularities at $\pi(\Sigma)$ (cf. [BS, 6]). Another point concerns the fact that the formula of $\nabla_{B S}$ in [BS] and [TTs] contains vector fields, kernel functions and forms. To justify applying $\bar{\partial}$ to such a sum, we find it convenient to present $\nabla_{B S}$ as the sum of a holomorphic action and a pure fiber integral of a form. This lends things easier to justification at the expense of slightly lengthened calculations.

As a by-product of (1) we obtain a precise description of the singularity of $\nabla_{B S}$ at the singular locus. This is based on an argument similar to that of [BB, 12c]. From this behavior of $\nabla_{B S}$ we obtain as a corollary [BB, Theorem 2.2] which describes the degeneration of Quillen metric near the singular locus. It is somewhat surprising now this degeneration is proved by local methods independent of the estimates on holomorphic torsion and Quillen metric that went into the proof of [BB, Theorem 2.1]. In [BB] it is mentioned that another proof of (1) for the Quillen connection can be derived using the isomorphism theorem of Deligne [D].

It should finally be remarked that (1) depends on the particular way a connection is chosen on $T Z$. We also calculate the curvature of a $\nabla_{B S}^{1}$ where the $\nabla_{T Z}$ is induced by a metric on $T X$ which is locally Euclidean at the singular points. In that case

$$
\frac{i}{2 \pi} \bar{\partial}_{S} \nabla_{B S}^{1}=\int_{X / S}\left[\operatorname{Td}\left(T Z, \nabla_{T Z}\right) \operatorname{ch}\left(E, \nabla_{E}\right)\right]_{2}+\frac{r k(E)}{6} \delta_{\pi(\Sigma)}
$$

Using (2) we immediately deduce the holonomy formula of [BB, Theorem 6.3]. We refer readers to $[\mathrm{BB}]$ for beautiful discussions of other applications of their main theorem, as well as thorough treatment of various background material.

In principle the methods utilized in this paper should extend to degenerate families of higher dimensional varieties by using the connection in [T] which is constructed from local parametrices. We hope to discuss this elsewhere.

\section{Trace Complex with Singularities}

We use the abbreviation f.s.o. to denote a holomorphic family of Riemann surfaces with ordinary singularities ([BB]). More precisely if $\pi: X \longrightarrow S$ is a proper surjective holomorphic map between complex manifolds such that the fiber $\pi^{-1}(s), s \in S$, is a reduced curve with only ordinary double points as singularities then the family of Riemann surfaces so parametrized is said to be an f.s.o.

It is well known ([BB, 3]) that for any $x \in X$ there exists local holomorphic coordinates $\left(z_{0}, z_{1}, \ldots, z_{n}\right)$ at $x$ and $\left(w_{1}, \ldots, w_{n}\right)$ at $s=\pi(x)$ such that $\pi$ is expressed by

$$
w_{i}=z_{i} \quad i=1, \ldots, n
$$

or by

$$
w_{1}=z_{0} z_{1}, \quad w_{i}=z_{l}, \quad i=2, \ldots, n .
$$

Thanks to the existence of such coordinates without loss of generality for purposes of discussing $(1,1)$ curvatures, it will be sufficient to take a coordinate slice, or that 
dimension of $S$ is one in (1.1). Since the subject matter of the discussion will be of local nature we can further assume that $S=\Delta_{\varepsilon_{0}}=\left\{z \in \mathbb{C}|| z \mid<\varepsilon_{0}\right\}$ and that $D=\pi^{-1}(0)$ is the only singular fiber with the set $\Sigma$ of ordinary double points.

Let $E \longrightarrow X$ be a holomorphic vector bundle, and $\lambda_{E}$ the Knudsen-Mumford determinant bundle associated to the direct image complex $R^{\cdot} \pi_{*} E$.

Let $\mathscr{T}_{X}$ be the tangent sheaf of $X, \mathscr{D}_{E}=\mathscr{D}_{\bar{E}}$ be the sheaf of holomorphic differential operators of order $\leqq 1$ acting on $E$, then the Atiyah algebra $\mathscr{A}_{E}$ is the subsheaf of $\mathscr{D}_{E}$ consisting of elements whose symbol is the identity. $\mathscr{T}_{\pi} \subset \mathscr{T}_{X}$ is the subalgebra of projectable vector fields, and we shall need the following notations. Let $\mathscr{T}_{S, \pi(D)} \subset \mathscr{T}_{S}$ be the subalgebra of vector fields that preserve $\pi(D)$ (cf. [BS, 6]). Let

$$
\mathscr{T}_{\pi \cdot D}=d \pi^{-1}\left(\mathscr{T}_{S, \pi(D)}\right) \subset \mathscr{T}_{\pi}
$$

be the projectable vector fields tangent to $D$, and

$$
\mathscr{T}_{X / S, D}=\left.\operatorname{ker} d \pi\right|_{\mathscr{T} \pi, D}
$$

Let $\varepsilon: \mathscr{D}_{E} \rightarrow \operatorname{End}(E) \otimes \mathscr{T}$ be the symbol map. Then

$$
\begin{aligned}
\mathscr{A}_{E, \pi}(\log D) & =\varepsilon^{-1}\left(\mathscr{T}_{\pi, D}\right) \subset \mathscr{A}_{E}, \\
\mathscr{A}_{E / S}(\log D) & =\varepsilon^{-1}\left(\mathscr{T}_{X / S, D}\right) \subset \mathscr{A}_{E, \pi}, \\
\mathscr{A}_{\lambda_{E}}(\log \pi(D)) & =\varepsilon^{-1}\left(\mathscr{T}_{S, \pi(D)}\right) \subset \mathscr{A}_{\lambda_{E}} .
\end{aligned}
$$

All of these are coherent analytic sheaves. Further define the following complexes with obvious differentials (inclusions):

$$
\mathscr{T}_{\pi, D}^{i}=\left\{\begin{array}{ll}
\mathscr{T}_{\pi, D} & i=0 \\
\mathscr{T}_{X / S, D}, & i=-1
\end{array} \quad \mathscr{A}_{E, \pi}^{l}(\log D)= \begin{cases}\mathscr{A}_{E, \pi}(\log D), & i=0 \\
\mathscr{A}_{E / S}(\log D), & i=-1 .\end{cases}\right.
$$

Lemma 1.1. Let $\pi: X \longrightarrow S$ be an f.s.o.

(i) The sequence

$$
0 \longrightarrow \mathscr{T}_{X / S, D} \longrightarrow \mathscr{T}_{\pi, D} \longrightarrow \mathscr{T}_{S, \pi(D)} \longrightarrow 0
$$

is exact, and we have $R^{0} \pi_{*} \mathscr{T}_{\pi, D} \cong R^{0} \pi_{*} \mathscr{A}_{E, \pi}(\log D) \cong \mathscr{T}_{S, \pi(D)}$.

(ii) For a given $p \in \Sigma$ and local coordinates $\left(z_{0}, z_{1}\right)$ at $p$ in $U$ such that $\pi\left(z_{0}, z_{1}\right)=z_{0} z_{1}=s$, then

$$
\mathscr{T}_{X / S, D}(U)=\left\{f\left(z_{0} \frac{\partial}{\partial z_{0}}-z_{1} \frac{\partial}{\partial z_{1}}\right) \mid f \in \mathcal{O}_{X}(U)\right\} .
$$

Proof. All assertions follow readily from the definitions and the formulas

$$
\pi_{*}\left(z_{0} \frac{\partial}{\partial z_{0}}\right)=\pi_{*}\left(z_{1} \frac{\partial}{\partial z_{1}}\right)=s \frac{\partial}{\partial s} .
$$

Lemma 1.2. Let $\pi: X \longrightarrow S$ be an f.s.o. Then $X \times_{S} X$ is a normal variety.

Proof. $X \times_{S} X$ is imbedded as an irreducible hypersurface in the smooth variety $X \times X$. Further the set of singularities of $X \times{ }_{S} X$ is exactly $\Sigma$, seated in the diagonal of $X \times_{S} X$, and hence $X \times_{S} X$ is a nonsingular in codimension 1. The assertion then follows from the standard criterion for normality (cf. [Mum.]). 
Let $\left(z_{0}, z_{1}, \zeta_{0}, \zeta_{1}\right)$ be local coordinates in $X \times X$, then the hypersurface $X \times_{S} X$ is given by

$$
z_{0} z_{1}-\zeta_{0} \zeta_{1}=0
$$

with $p \in \Sigma$ corresponding to the origin.

Let $\Delta: X \longrightarrow X \times_{S} X$ be the diagonal map, $\mathscr{I}(2 \Delta)$ be the ideal sheaf of $2 \Delta$ and $\mathcal{O}_{X \times{ }_{S} X}(2 \Delta)$ be the sheaf associated to the presheaf:

$$
U \mapsto\left\{f=\frac{g}{h} \mid g, h \in \mathcal{O}_{X \times{ }_{S} X}(U), v_{\Delta}(f) \geqq-2\right\}
$$

where $v_{\Delta}$ is the valuation at $\Delta$. Let $\omega=\omega_{X} \otimes \pi^{*} \omega_{S}^{-1}$ be the relative dualizing sheaf, where $\omega_{X}$ and $\omega_{S}$ are canonical sheaves on $X$ and $S$ respectively. Put $E^{\circ}=E^{*} \otimes \omega$ and define $E \otimes E^{\circ}(2 \Delta)=\pi_{1}^{*} E \otimes \pi_{2}^{*} E^{\circ} \otimes \mathcal{O}_{X \times{ }_{S} X}(2 \Delta)$, where $\pi_{i}$ 's are projections of $X \times_{S} X$ onto its factors. Put

$$
\left.E \bowtie E^{\circ}(2 \Delta)\right|_{2 \Delta}=E \bowtie E^{\circ}(2 \Delta) / E \bowtie E^{\circ}
$$

and denote by $r$ the natural map:

$$
\left.E \bowtie E^{\circ}(2 \Delta) \stackrel{r}{\rightarrow} E \bowtie E^{\circ}(2 \Delta)\right|_{2 \Delta} \longrightarrow 0
$$

Taking residue along $\Delta-\Sigma$ gives a canonical isomorphism outside $\Sigma$ :

$$
\left.E \bowtie E^{\circ}(2 \Delta)\right|_{2 \Delta} \cong \mathscr{D} \leqq 1 \text { E/S } .
$$

Lemma 1.3. There exists a sheaf homomorphism $\varphi$ :

$$
0 \longrightarrow \mathscr{D} \leqq 1 \text { E/S }\left.(\log D) \stackrel{\varphi}{\rightarrow} E \bowtie E^{\circ}(2 \Delta)\right|_{2 \Delta}
$$

such that $\varphi$ restricts to the preceding canonical isomorphism outside $\Sigma$.

Proof. Fix $p \in \Sigma$ and let $\left(z_{0}, z_{1}\right)$ be the coordinates in $U \ni p$ as in Lemma 1.1. It suffices to specify $\left.\varphi\right|_{U}$. Put $U_{0}=\left\{z_{0} \neq 0\right\} U_{1}=\left\{z_{1} \neq 0\right\}$ and choose coordinates adapted to the fibration $\pi: X \longrightarrow S$ :

$$
\left\{\begin{array}{l}
z=z_{0} \\
s=z_{0} z_{1}
\end{array} \quad \text { on } U_{0} ; \quad\left\{\begin{array}{l}
\widetilde{z}=z_{1} \\
\widetilde{s}=z_{0} z_{1}
\end{array} \quad \text { on } U_{1}\right. \text {. }\right.
$$

Then in $U_{0}$

$$
\begin{aligned}
& \frac{\partial}{\partial z}=\frac{\partial}{\partial z_{0}}-\frac{z_{1}}{z_{0}} \frac{\partial}{\partial z_{1}}, \\
& \frac{\partial}{\partial s}=\frac{1}{z_{0}} \frac{\partial}{\partial z_{1}},
\end{aligned}
$$

and there are similar formulas on $U_{1}$. Write $\zeta, \widetilde{\zeta}$ for the corresponding coordinates in the second factor of $X \times_{S} X$. 
On the vector part of $\mathscr{D} \frac{\leqq 1}{E / S}(\log D)(U)$ we define $\varphi$ to be

$$
\begin{aligned}
& z_{0} \frac{\partial}{\partial z_{0}}-z_{1} \frac{\partial}{\partial z_{1}} \stackrel{\varphi}{\rightarrow} \frac{z d \zeta}{(\zeta-z)^{2}} \bmod \left(E 区 E^{\circ}\right) \text { on } U_{0}, \\
& z_{0} \frac{\partial}{\partial z_{0}}-z_{1} \frac{\partial}{\partial z_{1}} \stackrel{\varphi}{\rightarrow} \frac{-\widetilde{z} d \widetilde{\zeta}}{(\widetilde{\zeta}-\widetilde{z})^{2}} \bmod \left(E 区 E^{\circ}\right) \text { on } U_{1} .
\end{aligned}
$$

To see that $\varphi$ is well defined on $U_{0} \cap U_{1}$ we have first of all as relative differentials

$$
\frac{d \widetilde{\zeta}}{\widetilde{\zeta}}=-\frac{d \zeta}{\zeta} \text { on } U_{0} \cap U_{1}
$$

( since $\frac{d \zeta_{0}}{\zeta_{0}}+\frac{d \zeta_{1}}{\zeta_{1}}=\pi^{*} \frac{d s}{s}$ ), furthermore by (1.2) it follows that

$$
\frac{\zeta_{0} z_{0}}{\left(\zeta_{0}-z_{0}\right)^{2}}=\frac{\zeta_{1} z_{1}}{\left(\zeta_{1}-z_{1}\right)^{2}} \in \mathcal{O}(2 \Delta)(U-p) \quad(=\mathcal{O}(2 \Delta)(U)) .
$$

We thus have

$$
\frac{z d \zeta}{(\zeta-z)^{2}} \in E \bowtie E^{\circ}(2 \Delta)(U) .
$$

Next on the endomorphism part of $\mathscr{D} \leqq 1 / S(\log D)(U)$, define $\varphi$ by

$$
\begin{gathered}
\Phi \stackrel{\varphi}{\rightarrow} \frac{\Phi d \zeta}{\zeta-z} \bmod \left(E \bowtie E^{\circ}\right) \quad \text { on } U_{0}, \\
\Phi \stackrel{\varphi}{\rightarrow} \frac{\Phi d \widetilde{\zeta}}{\widetilde{\zeta}-\widetilde{z}} \bmod \left(E \bowtie E^{\circ}\right) \quad \text { on } U_{1} .
\end{gathered}
$$

Again one has by (1.2)

$$
\frac{\zeta_{0}}{\zeta_{0}-z_{0}}=-\frac{z_{1}}{\zeta_{1}-z_{1}} \in \mathcal{O}(2 \Delta)(U)
$$

and it follows that on $U_{0} \cap U_{1}$

$$
\Phi \frac{d \widetilde{\zeta}}{\widetilde{\zeta}-\widetilde{z}}-\Phi \frac{d \zeta}{\zeta-z}=-\Phi \frac{d \zeta}{\zeta} \equiv 0 \bmod \left(E \otimes E^{\circ}\right) .
$$

For $\partial \in \mathscr{D}_{E / S}(\log D)$ along $\Delta-\Sigma$ we have $\operatorname{Res}_{\varphi}(\partial)=\partial$ by using (1.4). This completes the proof of the lemma.

Let $\operatorname{tr}:\left.E \bowtie E^{\circ}\right|_{\Delta} \longrightarrow \omega$ be the trace map. Via $\operatorname{tr}$ and $\varphi$ the exact sequence $0 \longrightarrow E \bowtie E^{\circ} / E \rrbracket E^{\circ}(-\Delta) \longrightarrow E \bowtie E^{\circ}(2 \Delta) /\left.E \bowtie E^{\circ}(-\Delta) \stackrel{\bar{r}}{\rightarrow} E \bowtie E^{\circ}(2 \Delta)\right|_{2 \Delta} \rightarrow 0$ is pushed into

$$
0 \longrightarrow \omega \longrightarrow{ }^{\operatorname{tr}} \mathscr{A}_{E}^{-1}(\log D) \stackrel{\text { Res }}{\longrightarrow} \mathscr{A}_{E / S}(\log D) \longrightarrow 0,
$$


where we define ${ }^{\operatorname{tr}} \mathscr{A}_{E}^{-1}(\log D)$ to be $\bar{r}^{-1}\left(\varphi\left(\mathscr{A}_{E / S}(\log D)\right)\right)$ modulo the traceless elements in $\operatorname{End}(E) \otimes \omega$. Now we can define the trace complex to be

$$
{ }^{\operatorname{tr}} \mathscr{A}_{E}^{i}(\log D)= \begin{cases}\mathscr{A}_{E, \pi}(\log D), & i=0 \\ { }^{t r} \mathscr{A}_{E}^{-1}(\log D), & i=-1\end{cases}
$$

with the differential given by Res.

Proposition 1.4. $R^{0} \pi_{*}\left({ }^{t r} \mathscr{A}_{E}(\log D)\right)$ is canonically isomorphic to $\mathscr{A}_{\lambda_{E}}(\log (\pi(D)))$. Proof. The proof follows [BS, Theorem 2.3.1]. Assume first that $R^{1} \pi_{*} E=0$. Put

$$
\begin{gathered}
\mathscr{B}_{E}(\log D)=r^{-1}\left(\varphi\left(A_{E / S}(\log D)\right)\right) \subset E \bowtie E^{\circ}(2 \Delta) \\
\operatorname{Cone}^{i}(\log D)= \begin{cases}\mathscr{A}_{E, \pi}(\log D), & i=0 \\
\mathscr{B}_{E}(\log D), & i=-1\end{cases}
\end{gathered}
$$

where $r$ and $\varphi$ are the maps in (1.3) and Lemma 1.3. Then taking direct image of the exact sequence

$$
0 \longrightarrow E \bowtie E^{\circ}[1] \longrightarrow \text { Cone } \cdot(\log D) \rightarrow \mathscr{A}^{\cdot}{ }_{E, \pi}(\log D) \rightarrow 0
$$

one gets

$$
0 \longrightarrow \operatorname{End}\left(\pi_{*} E\right) \longrightarrow R^{0}(\pi \times \pi)_{*}(\text { Cone } \cdot(\log D)) \longrightarrow \mathscr{T}_{S, \pi(D)} \longrightarrow 0 .
$$

One can then define action of $R^{0}(\pi \times \pi)_{*}\left(\right.$ Cone $\left.^{*}(\log D)\right)$ on $\pi_{*} E$ by using Cousin resolution of Cone $(\log D)$ as in [BS], noting that the action is well defined outside $\Sigma$ and can be extended (uniquely) across $\Sigma$ since $\Sigma$ is of codimension 2 in $X$. Therefore one concludes that

$$
R^{0}(\pi \times \pi)_{*} \text { Cone } \cdot(\log D) \cong A_{\pi_{*} E}(\log (\pi(D)))
$$

and upon taking traces one gets Proposition 1.4 in the case $R^{1} \pi_{*} E=0$.

The general case follows from above by considering

$$
\left.0 \longrightarrow E(-Z) \longrightarrow E \longrightarrow i_{*} E\right|_{Z} \longrightarrow 0
$$

where $Z \stackrel{i}{\rightarrow} X$ is a divisor étale over $S$ and $Z \cap \Sigma=\phi$. Put $E_{Z}=\left(\left.\pi\right|_{Z}\right)_{*}\left(\left.E\right|_{Z}\right)$, one has the isomorphism

$$
R^{0} \pi_{*}\left({ }^{t r} \mathscr{A}_{E}(\log D)\right)=R^{0} \pi_{*}\left({ }^{t r} \mathscr{A}_{E(-Z)}(\log D)\right)+\mathscr{A}_{\operatorname{det} E_{Z}}(\log \pi(D)) .
$$

For further details we refer to [BS].

Remark. We have not pursued the Lie algebra structure on ${ }^{\operatorname{tr}} \mathscr{A}^{\cdot}(\log D)$ in this paper. This would require the introduction of one more term in the complex as in [BS].

\section{Beilinson Schechtman Connection for Degenerate Families}

We shall first put the Beilinson Schechtman connection in a simpler complex than that described in [TTs, 1]. Starting with smooth families $\pi: X \longrightarrow S$, the push 
forward of

$$
0 \longrightarrow \mathcal{O}_{S} \longrightarrow \mathscr{A}_{\lambda_{E}} \longrightarrow \mathscr{T}_{S} \longrightarrow 0
$$

by $\mathcal{O}_{S} \longrightarrow C_{S}$ gives

$$
0 \longrightarrow C_{S} \longrightarrow \mathscr{A}_{\lambda_{E}} \longrightarrow \mathscr{T}_{S} \longrightarrow 0
$$

whose splittings correspond to $(1,0)$ connections on $\lambda_{E}$. The cohomology

$$
R^{0} \pi_{*}\left({ }^{t r} \mathscr{A}_{E} \otimes_{C_{S}} C_{S}\right) \cong \mathscr{A}_{i_{E}} \otimes_{C_{S}} C_{S}
$$

can be computed using the $\bar{\partial}_{z}$ ( $\bar{\partial}$ along fibers) resolution of ${ }^{t r} \mathscr{A}^{\cdot}{ }_{E} \otimes_{\mathcal{C}_{S}} C_{S}$ and is represented by

$$
\left\{(\tau+\eta) \mid \tau \in \mathscr{A}_{E, \pi} \otimes \Omega^{0,0}(X), \quad \eta \in{ }^{t r} \mathscr{A}_{E}^{-1} \otimes \Omega^{0,1}(X), \bar{\partial}_{z} \tau=\operatorname{Res} \eta\right\}
$$

modulo the subset

$$
\left\{(\tau+\eta) \mid \tau=\operatorname{Res} \psi, \eta=\bar{\partial}_{z} \psi, \text { for some } \psi \in{ }^{t r} \mathscr{A}_{E}^{-1} \otimes \Omega^{0,0}(X)\right\},
$$

where $\Omega^{p, q}$ is the sheaf of bigraded relative smooth forms.

Given $C^{\infty}$ connections $\nabla_{E}, \nabla_{T}$ on $X$ for $E$ and $T_{X / S}$ we have the expressions in local coordinates

$$
\nabla_{T, \frac{\partial}{\partial z}}=\frac{\partial}{\partial z}+\Phi_{T} d z \quad \nabla_{E, \frac{\partial}{\partial z}}=\frac{\partial}{\partial z}+\Phi_{E} d z
$$

where $z$ is a holomorphic coordinate along the fiber. We put

$$
\begin{aligned}
& \bar{C}\left(\nabla_{E}\right)=\bar{\partial}\left(\Phi_{E} d z\right) \in \Omega_{X}^{0,1} \otimes \Omega^{1,0}(\operatorname{End}(E)), \\
& \widetilde{C}\left(\nabla_{E}\right)=\bar{\partial}_{z}\left(\Phi_{E} d z\right) \in \Omega^{1,1}(\operatorname{End}(E)), \\
& \bar{c}_{1}\left(\nabla_{E}\right)=\frac{i}{2 \pi} \operatorname{tr} \bar{C}\left(\nabla_{E}\right) \in \Omega_{X}^{0,1} \otimes \Omega^{1,0} \\
& \widetilde{c}_{1}\left(\nabla_{E}\right)=\frac{i}{2 \pi} \operatorname{tr} \widetilde{C}\left(\nabla_{E}\right) \in \Omega^{1,1}
\end{aligned}
$$

and similar notations for $\nabla_{T}$. Let $v=\frac{\partial}{\partial s}$ be a local holomorphic vector field on $S$ and let

$$
\widetilde{v}=\frac{\partial}{\partial s}+a \frac{\partial}{\partial z}+B \in \mathscr{A}_{E, \pi} \otimes \Omega^{0,0}(X)
$$

be any $C^{\infty}$ lifting of $v$. Further set

$$
\begin{aligned}
A_{0} & =\frac{1}{2} \Phi_{T}+\Phi_{E}, \\
A_{1} & =\frac{1}{6} \Phi_{T}^{\prime}-\frac{1}{12} \Phi_{T}^{2}-\frac{1}{2} \Phi_{T} \Phi_{E}+\frac{1}{2} \Phi_{E}^{\prime}-\frac{1}{2} \Phi_{E}^{2}, \\
\pi_{\nabla_{E}}(\widetilde{v}) & =\widetilde{v}-\nabla_{E, \varepsilon(\widetilde{v})} .
\end{aligned}
$$


Now the Beilinson Schechtman connection is expressed as follows:

$$
\begin{aligned}
\nabla_{B S, v} & =[\tau+\eta] \in R^{0} \pi_{*}\left({ }^{t r} \mathscr{A}_{E} \otimes C_{S}\right), \\
\tau & =\widetilde{v} \in \mathscr{A}_{E, \pi} \otimes \Omega^{0,0}(X), \\
\eta & =p^{-1}\left(\overline{\partial_{z}} \widetilde{v}\right)+p^{0}(\widetilde{v}) \in{ }^{t r} \mathscr{A}_{E}^{-1} \otimes \Omega^{0,1}(X),
\end{aligned}
$$

where in local coordinates

$$
\begin{aligned}
p^{-1}\left(\overline{\partial_{z}} \widetilde{v}\right)= & \frac{1}{2 \pi i}\left\{\frac{\overline{\partial_{z}} a}{(\zeta-z)^{2}}+\frac{\overline{\partial_{z}} B}{\zeta-z}+\operatorname{tr}\left[\bar{\partial}_{z} B A_{0}+\bar{\partial}_{z} a A_{1}\right]\right\} d \zeta, \\
p^{0}(\widetilde{v})= & -\nabla_{\omega, \varepsilon(\widetilde{v})}\left(\frac{r k(E)}{6} \widetilde{c}_{1}\left(\nabla_{T}\right)+\frac{1}{2} \widetilde{c}_{1}\left(\nabla_{E}\right)\right) \\
& -\operatorname{tr}\left[\pi_{\nabla_{E}}(\widetilde{v})\left(\frac{1}{2} \widetilde{C}\left(\nabla_{T}\right)+\widetilde{C}\left(\nabla_{E}\right)\right)\right] .
\end{aligned}
$$

$\nabla_{B S, v}$ is independent of the lifting $\widetilde{v}$ of $v$.

Remark. For later purpose we note that $p^{-1}\left(\overline{\partial_{z}} \widetilde{v}\right)$ is the image of $p^{-1}(\bar{\partial} \widetilde{v})$ via the projection induced by $\Omega_{X}^{0,1} \longrightarrow \Omega_{X / S}^{0,1}$. Similarly in $p^{0}(\widetilde{v})$ with all tildas replaced by bars one gets an element of ${ }^{t r} \mathscr{A}_{E}^{-1} \otimes \Omega_{X}^{0,1}(X)$ also denoted by $p^{0}(\widetilde{v})$ whose image in ${ }^{\operatorname{tr}} \mathscr{A}_{E}^{-1} \otimes \Omega^{0,1}$ is the above $p^{0}(\widetilde{v})$.

To get holomorphic connections we use Čech complexes so that $R^{0} \pi_{*}\left({ }^{t r} \mathscr{A}^{\cdot}{ }_{E}\right)$ is represented by

$$
\left\{\left(c^{0}+c^{1}\right) \mid c^{0} \in C^{0}\left(\mathscr{U}, \mathscr{A}_{E, \pi}\right), \quad c^{1} \in C^{1}\left(\mathscr{U}^{t r} \mathscr{A}_{E}^{-1}\right), \delta c^{0}=\operatorname{Res} c^{1}, \delta c^{1}=0\right\}
$$

modulo the subset

$$
\left\{\left(c^{0}+c^{1}\right) \mid c^{0}=\operatorname{Res} \widetilde{c}^{0}, c^{1}=\delta \widetilde{c}^{0}, \text { for some } \widetilde{c}^{0} \in C^{0}\left(\mathscr{U},{ }^{t r} \mathscr{A}_{E}^{-1}\right)\right\},
$$

where $\mathscr{U}=\left\{U_{\alpha}\right\}$ is a relative Stein covering of $X$ and $\delta$ is the Čech coboundary. Let $\left\{\rho_{\alpha}\right\}$ be a partition of 1 subordinated to $\mathscr{U}$, then the maps

$$
\rho\left(c^{0}\right)=\sum_{\alpha} \rho_{\alpha} c_{\alpha}^{0} \quad \rho\left(c^{1}\right)=\sum_{\alpha, \beta} \rho_{\beta} \overline{\partial_{z}} \rho_{\alpha} c_{\alpha \beta}^{1}
$$

embed the Čech complexes into the $\overline{\partial_{z}}$ complexes and induce a map in cohomology:

$$
\rho: R^{0} \pi_{*}\left({ }^{t r} \mathscr{A} \cdot{ }_{E}\right) \longrightarrow R^{0} \pi_{*}\left({ }^{t r} \mathscr{A}_{E}\right) \otimes \mathscr{C}_{S} .
$$

Now let

$$
[\tau+\eta] \in \mathscr{C}_{\lambda_{\lambda_{E}}}, \quad c=\left[c^{0}+c^{1}\right] \in \mathscr{A}_{\lambda_{E}}
$$

be liftings of $v=\frac{\partial}{\partial S}$. Since $\tau-\rho\left(c^{0}\right) \in \mathscr{A}_{E / S} \otimes \Omega^{0,0}(X)$ there exists $\psi \in{ }^{\operatorname{tr}} \mathscr{A}_{E}^{-1} \otimes$ $\Omega^{0,0}(X)$ such that

$$
\operatorname{Res} \psi=\tau-\rho\left(c^{0}\right) .
$$

Similarly

$$
\gamma=\eta-\rho\left(c^{1}\right)-\overline{\partial_{z}} \psi \in \Omega^{1,1}(X),
$$


since $\operatorname{Res} \gamma=0$. Combining (2.6) and (2.7) we have

$$
\tau+\eta=\gamma+\rho(c)+\operatorname{Res} \psi+\overline{\partial_{z}} \psi
$$

and therefore in cohomology:

$$
[\tau+\eta]=\int_{X / S} \gamma+[\rho(c)] \in \mathscr{C} \mathscr{A}_{\lambda_{E}} .
$$

We shall apply this to the case $[\tau+\eta]=\nabla_{B S, v}$.

Lemma 2.1. Let $c=\left[c^{0}+c^{1}\right]$ be a holomorphic lifting of $v=\frac{\partial}{\partial S}$ in $\mathscr{A}_{\lambda_{E}}$, then

$$
\nabla_{B S, v}=\int_{X / S}\left(p^{0}(\widetilde{v})+p^{-1}(\bar{\partial} \widetilde{v})-\rho\left(c^{1}\right)-\bar{\partial} \psi\right)+[\rho(c)]
$$

Remark. This representation of $\nabla_{B S, v}$ with the inclusion of a $c$ and $\psi$ appears to be more complicated than that in [TTs, (1.10)] and [BS, 5]. However, the latter expressions are hard to work with when one tries to compute the $(1,1)$ curvature as they combine both forms and vector fields. Lemma 2.1 expresses $\nabla_{B S, v}$ clearly as the sum of the holomorphic action $[\rho(c)]$ and the fiber integral of a smooth form.

Proposition 2.2. (Grothendieck Riemann Roch for smooth families).

$$
\left.\frac{i}{2 \pi} \overline{\partial_{S}} \nabla_{B S, v}=v\right\rfloor \int_{X / S}\left[\operatorname{Td}\left(T_{X / S}, \nabla_{T}\right) \operatorname{ch}\left(E, \nabla_{E}\right)\right]_{2}
$$

Proof. By Lemma 2.1 and the fact that $\bar{\partial} \rho\left(c^{1}\right)= \pm \rho\left(\delta c^{1}\right)=0$,

$$
\overline{\partial_{S}} \nabla_{B S, v}=\int_{X / S} \bar{\partial}\left(p^{0}(\widetilde{v})+p^{-1}(\bar{\partial} \widetilde{v})\right) .
$$

The calculation then proceeds as in [BS, p. 685].

We now come to degenerate families. As in Sect. 1 , let $\Delta_{\varepsilon_{0}}=\left\{s \in \mathbb{C}|| s \mid<\varepsilon_{0}\right\}$, $\pi: X \rightarrow \Delta_{\varepsilon_{0}}$ be an f.s.o. Let $Z_{s}=\pi^{-1}(s)$ and $Z_{0}=D$ is the only singular fiber. $\Sigma=\left\{p_{1}, \ldots, p_{n}\right\} \subset Z_{0}$ denotes the set of singular points. The local discussions will be the same at all points of $\Sigma$ so we just consider $p=p_{1}$. Employing again the local coordinates $\left(z_{0}, z_{1}\right)$ in neighborhood $U$ of $p$ we choose a particular $C^{\infty}$ lifting of $v=\frac{\hat{c}}{\hat{c} s}$ in $U-p$ :

$$
v_{0}=\frac{\overline{z_{1}}}{|z|^{2}} \frac{\partial}{\partial z_{0}}+\frac{\overline{z_{0}}}{|z|^{2}} \frac{\partial}{\partial z_{1}},
$$

where $|z|^{2}=\left|z_{0}\right|^{2}+\left|z_{1}\right|^{2} \cdot \pi_{*}\left(v_{0}\right)=\frac{\hat{c}}{\hat{c} s}$ follows from $\pi_{*}\left(\frac{\hat{c}}{\hat{c} z_{0}}\right)=z_{1} \frac{\hat{c}}{\hat{c} s}$ and $\pi_{*}\left(\frac{\hat{c}}{\partial z_{1}}\right)=$ $z_{0} \frac{\hat{c}}{\hat{\imath} s}$. Differentiating (2.8)

$$
\bar{\partial} v_{0}=\frac{\overline{z_{0}} d \overline{z_{1}}-\overline{z_{1}} d \overline{z_{0}}}{|z|^{4}}\left(z_{0} \frac{\partial}{\partial z_{0}}-z_{1} \frac{\partial}{\partial z_{1}}\right)
$$

which checks that $\bar{\partial} v_{0} \in \mathscr{A}_{E / S} \otimes \Omega^{0,1}$, i.e. the vector part is tangent to fibers. Let $\nabla_{\omega}$ and $\nabla_{E}$ be $C^{\infty}(1,0)$ connections on $\omega_{X / S}$ and $E, \nabla_{\omega}$ induces a $C^{\infty}$ connection $\nabla_{\omega^{-1}}$ on $T_{Z}$ in $X-\Sigma$. In terms of $\nabla_{\omega^{-1}}$ and $\nabla_{E}$ we have the formulas (2.3) (2.5). We proceed to estimate their singularities in $U$ using the coordinates in the open subset $U_{0}$ (Lemma 1.1). We also choose $\widetilde{v}=\nabla_{E, v_{0}}$ so that $\pi_{\nabla E}(\widetilde{v})=0$. 
Lemma 2.3. The following estimates are valid in $U$ :

(i) $\overline{\bar{\partial}} \widetilde{v}=\bar{\partial} v_{0}+\frac{\xi_{1}}{|z|^{4}}, \quad \xi_{1} \in \Omega^{0,1}(\operatorname{End}(E))(U)$,

(ii) $A_{0}=\frac{\xi_{2}}{z_{0}}, \quad \quad \xi_{2} \in C^{\infty}(U)$,

(iii) $A_{1}=\frac{1}{12 z_{0}^{2}}+\frac{\xi_{3}}{z_{0}^{2}}, \quad \xi_{3} \in C^{\infty}(U)$,

(iv) $p^{0}(\widetilde{v})=\frac{\xi_{4}}{|z|^{4}} \frac{d z_{0}}{z_{0}}, \quad \xi_{4} \in \Omega^{0,1}(U)$,

where

$$
\begin{aligned}
& \left|\xi_{1}\right| \leqq O\left(|z|^{2}\right), \quad\left|\xi_{2}\right| \leqq O(|z|), \\
& \left|\xi_{3}\right| \leqq O(|z|), \quad\left|\xi_{4}\right| \leqq O\left(|z|^{3}\right) .
\end{aligned}
$$

Proof. By our choice of $\widetilde{v}$

$$
\left.\bar{\partial} \widetilde{v}=\nabla_{E, \bar{\partial}_{\iota_{0}}}-v_{0}\right\rfloor C(E),
$$

(i) then follows from (2.9). We next consider (iii). Let $\theta$ be the smooth connection 1 form in $U$ :

$$
\nabla_{\omega}\left(\frac{d z_{0}}{z_{0}}\right)=\theta \frac{d z_{0}}{z_{0}}
$$

then acting on the section $d z_{0}$ we have by a gauge change

$$
\nabla_{\omega}\left(d z_{0}\right)=\left(\theta+\frac{d z_{0}}{z_{0}}\right) \otimes d z_{0}
$$

By the duality of $d z_{0}=d z$ and $\frac{\partial}{\partial z}=\frac{\partial}{\partial z_{0}}-\frac{z_{1}}{z_{0}} \frac{\partial}{\partial z_{1}}$ we have

$$
\nabla_{\omega-1}\left(\frac{\partial}{\partial z}\right)=\left(-\theta-\frac{d z_{0}}{z_{0}}\right) \otimes \frac{\partial}{\partial z} \text {. }
$$

Thus with respect to the frame $\frac{\partial}{\partial z}$ in $U$

$$
\Phi_{T}=-f-\frac{1}{z_{0}},
$$

where $\left.f=\frac{\hat{c}}{\partial z}\right\rfloor \theta$ and

$$
\Phi_{T}^{\prime}=\left(\frac{\partial}{\partial z_{0}}-\frac{z_{1}}{z_{0}} \frac{\partial}{\partial z_{1}}\right)\left(-f-\frac{1}{z_{0}}\right)=\frac{1}{z_{0}^{2}}-f_{z} .
$$

Clearly $|f| \leqq O\left(\frac{|z|}{\left|z_{0}\right|}\right),\left|f_{z}\right| \leqq O\left(\frac{|z|}{\left|z_{0}\right|^{2}}\right)$ and

$$
\frac{1}{6} \Phi_{T}^{\prime}-\frac{1}{12} \Phi_{T}^{2}=\frac{1}{12} \frac{1}{z_{0}^{2}}+\frac{\alpha}{z_{0}^{2}},
$$


where $|\alpha| \leqq O(|z|)$. In the same way we have

$$
\Phi_{E}=\frac{\beta}{z_{0}} \quad \text { where }|\beta| \leqq O(|z|) .
$$

The assertion (iii) now follows from the formula of $A_{1}$ in (2.3). These arguments also immediately yield the simpler case (ii). Finally to verify (iv) note that

$$
\bar{\partial}\left(\Phi_{T} d z\right)=g \frac{d z_{0}}{z_{0}},
$$

where $g$ is a smooth form and

$$
\nabla_{\omega, v_{0}}\left(g \frac{d z_{0}}{z_{0}}\right)=\left(\operatorname{Lie}\left(v_{0}\right) g\right) \frac{d z_{0}}{z_{0}}+g \nabla_{\omega, v_{0}}\left(\frac{d z_{0}}{z_{0}}\right) .
$$

This result then follows since $v_{0}$ has singularity of order 1 .

Now in the expansion (2.5) of $p^{-1}(\bar{\partial} \widetilde{v})$ we restrict the zero order term to the diagonal (in $X \times_{S} X$ ) and consider its most singular part at $p \in U$.

Corollary 2.4. Let $\widetilde{v}=\nabla_{E, v_{0}}$ then we have on $U$

$$
\operatorname{tr}\left\{\bar{\partial}_{z} B A_{0}+\bar{\partial}_{z} a A_{1}\right\} d z_{0}=\frac{r k(E)}{12} \cdot \frac{\overline{z_{0}} d \overline{z_{1}}-\overline{z_{1}} d \overline{z_{0}}}{|z|^{4}} \cdot \frac{d z_{0}}{z_{0}}+\frac{\xi}{|z|^{4}} \frac{d z_{0}}{z_{0}}
$$

where $\xi \in \Omega^{0,1}(U)$ and $|\xi| \leqq O\left(|z|^{2}\right)$.

Proof. From (2.9) and Lemma 2.3 (i)

$$
\bar{\partial} a=\frac{\overline{z_{0}} d \overline{z_{1}}-\overline{z_{1}} d \overline{z_{0}}}{|z|^{4}} \cdot z_{0}
$$

and

$$
\bar{\partial}_{z} B=\frac{\xi_{1}}{|z|^{4}} \quad \text { with }\left|\xi_{1}\right| \leqq O\left(|z|^{2}\right) .
$$

The corollary then follows by the estimates in Lemma 2.3 (ii) (iii).

\section{Curvature of Determinant Bundle in Degenerate Case}

As in Sect. 2 let $\pi: X \rightarrow \Delta_{\varepsilon}$ be an f.s.o. with $\pi^{-1}(0)=Z_{0}=D$ the only singular fiber, $\Sigma$ the singular set in $Z_{0}$. Let $\nabla_{\omega}$ and $\nabla_{E}$ be $C^{\infty}(1,0)$ connections on $\omega_{X / S}$ and $E . \nabla_{\omega}$ induces a $C^{\infty}$ connection $\nabla_{\omega^{-1}}$ on $T_{Z}$ in $X-\Sigma$. Let $\nabla_{B S}$ be the Beilinson-Schechtman connection of $\lambda_{E}$ associated to $\nabla_{\omega^{-1}}$ and $\nabla_{E}$ on $\Delta_{\varepsilon}-\{0\}$.

Theorem 3.1. With notations as above $\nabla_{B S}$ extends as an $L^{1}$ connection of $\lambda_{E}$ over $\Delta_{\varepsilon}$ and its curvature computed in the sense of currents is given by

$$
\frac{i}{2 \pi} \overline{\partial_{S}} \nabla_{B S}=\int_{X / S}\left[\operatorname{Td}\left(T_{Z}, \nabla_{\omega^{-1}}\right) \operatorname{ch}\left(E, \nabla_{E}\right)\right]_{2}+\frac{n r k(E)}{12} \delta,
$$

where $\delta$ is the delta function supported at 0 and $n$ is the number of singular points in $\Sigma$. 
Since our local arguments are the same at all the points of $\Sigma$, for simplicity we assume that $\Sigma$ consists of one point $p$. We choose a Stein covering $\mathscr{U}=\left\{U_{\alpha}\right\}$ of $X$ such that $p \in U_{\alpha_{0}}$ and $p \notin U_{\alpha}$ if $\alpha \neq \alpha_{0} .\left\{p_{\alpha}\right\}$ is a partition of 1 subordinated to $\mathscr{U}$. Let

$$
\left(\widetilde{c}^{0}+\widetilde{c}^{1}\right) \in C \cdot\left(\mathscr{U}^{t r} \mathscr{A}_{E}(\log D)\right)
$$

be a cocycle such that $\pi_{*}\left(\varepsilon\left(\widetilde{c}^{0}\right)\right)=s \frac{\partial}{\partial_{s}}$. Put

$$
c^{0}=\frac{1}{s} \widetilde{c}^{0}, \quad c^{1}=\frac{1}{s} \widetilde{c}^{1}, \quad c=c^{0}+c^{1} .
$$

We may assume that $c_{\alpha}^{0}$ is holomorphic in $U_{\alpha}$ if $\alpha \neq \alpha_{0} . \rho\left(c^{1}\right)$ are defined as before. $\left(z_{0}, z_{1}\right)$ and $(z, s)$ are the coordinates in Lemma 1.1. On $U_{\alpha_{0}}$ let $\widetilde{v}=\nabla_{E, v_{0}}, v_{0}$ as in (2.8) and

$$
\widetilde{\psi}_{\alpha_{0}}=\left[\frac{\widetilde{a}}{(\zeta-z)^{2}}+\frac{\widetilde{B}}{(\zeta-z)}\right] d \zeta \in^{t r} \mathscr{A}_{E}^{-1}(\log D) \otimes \Omega^{0,0}
$$

be a smooth lifting of $s \tilde{v}-\rho\left(\widetilde{c}^{0}\right)$ (outside $p$ ). For $\alpha \neq \alpha_{0}$ choose $\psi_{\alpha}$ to be any smooth lifting of $\widetilde{v}-\left.\rho\left(c^{0}\right)\right|_{U_{x}}$. Set

$$
\psi_{x_{0}}=\frac{1}{s} \widetilde{\psi}_{\alpha_{0}}, \quad \psi=\sum_{\alpha} \rho_{\alpha} \psi_{\alpha}
$$

Lemma 3.2. On $U_{x_{0}}$,

$$
\psi=\left(\frac{\widetilde{a}}{(\zeta-z)^{2}}+\frac{\widetilde{B}}{\zeta-z}+\frac{\breve{\zeta}_{5}}{z_{0}}\right) d z_{0} \in^{t r} \mathscr{A}_{E}^{-1}(\log D) \otimes \Omega^{0,0}, \quad \text { where } \quad \xi_{5} \in
$$
$C^{\infty}\left(U_{x_{0}}\right)$ and $\xi_{5}=0$ in a neighborhood of $p$.

(ii) $s \rho\left(c^{1}\right)=\left(\frac{\mu_{-1}}{(\zeta-z)^{2}}+\frac{\mu_{0}}{\zeta-z}+\frac{\mu_{1}}{z_{0}}\right) d z_{0} \in{ }^{t r} \mathscr{A}_{E}^{-1}(\log D) \otimes \Omega^{0,0}$, where $\mu_{1} \in C^{\infty}\left(U_{\alpha_{0}}\right)$ and $\mu_{1}=0$ in a neighborhood $W$ of $p$.

Proof. (i) $\sum_{\beta \neq x_{0}} \rho_{\beta} \psi_{\beta}$ is smooth and vanishes in a neighborhood of $p$. The possible pole in $\frac{\xi_{5}}{z_{0}}$ arises from the fact that if local expressions are given in $U_{1}$ coordinates (cf. Lemma 1.1) and one transforms it to $U_{0}$ coordinates via $z_{1}=\frac{s}{z_{0}}, \widetilde{s}=s$, then the gauge change formulas of [BS, p. 683] show a possible factor $\frac{1}{z_{0}}$. (ii) The proof can be done in the similar way as (i).

We now prove that $\nabla_{B S}$ is $L^{1}$. Since $[\rho(\widetilde{c})]$ gives a class in $\mathscr{A}_{i_{E}}(\log \pi(D))$ and therefore $[\rho(c)]$ is $L^{1}$ it suffices to show, in view of Lemma 2.1, that

$$
I(q)=\int_{B_{\varepsilon}(q)}\left|\left(p^{0}(\widetilde{v})+p^{-1}(\bar{\partial} \widetilde{v})-\rho\left(c^{1}\right)-\bar{\partial} \psi\right) d s \wedge d \bar{s}\right|<\infty
$$

for all points $q \in D$, where $B_{\varepsilon}(q)$ is an $\varepsilon$-ball centered at $q$. For $q \notin U_{\alpha_{0}}$, we have

$$
I(q) \leqq \text { const. } \int_{B_{\varepsilon}(q)}\left|\frac{\beta_{1}}{s} \wedge d s \wedge d \bar{s}\right|<\infty,
$$

where $\beta_{1} \in \Omega_{X}^{1,1}\left(B_{\varepsilon}(q)\right)$ because $p^{0}(\widetilde{v}), \bar{\partial} \psi$ and $p^{-1}(\bar{\partial} \widetilde{v})$ are smooth at $q$. Note that $d s=z_{0} d z_{1}+z_{1} d z_{0}$ so that

$$
\frac{d z_{0}}{z_{0}} \wedge d s \wedge d \bar{s}=d z_{0} \wedge d z_{1} \wedge d \bar{s} .
$$


Using this, Lemma 3.2, Corollary 2.4 we have for $q \in U_{\alpha_{0}} q \neq p$ :

$$
I(q) \leqq \text { const. } \int_{B_{\varepsilon}(q)}\left|\frac{\beta_{2}}{s} \wedge d z_{0} \wedge d z_{1} \wedge d \bar{s}\right|<\infty,
$$

where $\beta_{2} \in \Omega_{X}^{0,1}\left(B_{\varepsilon}(q)\right)$, and for $q=p$ :

$$
I(q) \leqq \text { const. } \int_{B_{\varepsilon}(q)}\left|\frac{1}{|z|^{3}} \wedge d z_{0} \wedge d \overline{z_{0}} \wedge d z_{1} \wedge d \overline{z_{1}}\right|<\infty .
$$

Thus (3.1) follows from (3.2)-(3.4).

We now compute $\bar{\partial}_{S} \nabla_{B S}$ in the sense of currents. First of all since $C\left(\nabla_{\omega^{-1}}\right)$ and $C(E)$ are smooth on $X$, the fiber integrals in Theorem 3.1 exist as currents on $S$ by [BB, 2(a)] (it follows from the proof of Proposition 4.1 that the singularity at 0 is no worse than $\log |s|)$. Let $v=\frac{\partial}{\partial S}$ and $\varphi=\varphi(s) d s$ be a $(1,0)$ smooth form with compact support on $\Delta_{\varepsilon_{0}}$. Then the distributional derivative $\bar{\partial}_{S} \nabla_{B S}$ is given by

$$
\overline{\partial_{S}} \nabla_{B S, v}(\varphi(s) d s)=-\int_{S} \nabla_{B S, v}\left(\bar{\partial}_{S} \varphi\right)=-\int_{X} \nabla_{B S, v} \wedge \bar{\partial} \pi^{*} \varphi
$$

where $\nabla_{B S, v}$ in (3.5) stands for its formula in Lemma 2.1 without the fiber integral. Let $T_{\varepsilon}(D)$ be an $\varepsilon$-tubular neighborhood of $D$ in $X$, then by the fact that $\nabla_{B S}$ is $L^{1}$

$$
\begin{aligned}
& -\int_{X} \nabla_{B S, v} \wedge \bar{\partial} \pi^{*} \varphi=-\lim _{\varepsilon \rightarrow 0} \int_{X-T_{\varepsilon}(D)} \nabla_{B S, v} \wedge \bar{\partial} \pi^{*} \varphi \\
& =-\lim _{\varepsilon \rightarrow 0} \int_{X-T_{\varepsilon}(D)} \bar{\partial}\left\{\nabla_{B S, v} \wedge \pi^{*} \varphi\right\}+\lim _{\varepsilon \rightarrow 0} \int_{X-T_{\varepsilon}(D)}\left(\bar{\partial} \nabla_{B S, v}\right) \wedge \pi^{*} \varphi \\
& \left.=\lim _{\varepsilon \rightarrow 0} \int_{\partial T_{t}(D)} \nabla_{B S, v} \wedge \pi^{*} \varphi+\frac{2 \pi}{i} \int_{S}\{v\rfloor \int_{X / S}\left[\operatorname{Td}\left(T_{X / S}, \nabla_{\omega^{-1}}\right) \operatorname{ch}\left(E, \nabla_{E}\right)\right]_{2}\right\} \wedge \varphi,
\end{aligned}
$$

where in $X-T_{\varepsilon}(D), \bar{\partial} \nabla_{B S, v}$ is its usual derivative hence the last term in (3.6) follows from Proposition 2.2.

\section{Lemma 3.3.}

$$
\lim _{\varepsilon \rightarrow 0} \int_{\partial T_{\varepsilon}(D)} \nabla_{B S, v} \wedge \pi^{*} \varphi=\lim _{\varepsilon \rightarrow 0} \int_{\partial B_{\varepsilon}(p)}\left(p^{0}(\widetilde{v})+p^{-1}(\bar{\partial} \widetilde{v})-\bar{\partial} \psi_{x_{0}}\right) \varphi(s) d s
$$

Assuming Lemma 3.3 for the moment we use (2.5), Corollary 2.4, Lemma 2.3 and Lemma 3.2 to evaluate its right-hand side, and we obtain

$$
\lim _{\varepsilon \rightarrow 0} \frac{2 \pi}{i} \int_{\partial B_{\varepsilon}(p)}\left(\frac{r k(E)}{12} \omega^{0} \varphi(s)+\frac{\beta \varphi(s)}{|z|^{4}} d z_{0} \wedge d z_{1}\right)=\frac{2 \pi}{i} \frac{r k(E)}{12} \varphi(0)
$$

where $\omega^{0}=\frac{-1}{(2 \pi i)^{2}} \frac{\overline{z_{0}} d \overline{z_{1}}-\overline{z_{1}} d \overline{z_{0}}}{|z|^{4}} d z_{0} \wedge d z_{1}$ is the Bochner kernel in dimension 2, and $\beta \in \Omega^{0,1}\left(U_{\alpha_{0}}\right),|\beta| \leqq O\left(|z|^{2}\right)$. Theorem 3.1 thus follows from (3.6) and (3.7). 
We turn now to the proof of Lemma 3.3. Let $W$ be the neighborhood of $p$ given in Lemma 3.2 (ii). Let $\chi \in C_{0}^{\infty}(W)$ be such that $0 \leqq \chi \leqq 1$, and $\chi \equiv 1$ in a smaller neighborhood $W_{1}$ of $p$. Let $\Phi(s)$ denote the connection matrix of $[\rho(c)]$ and $\gamma=$ $p^{0}(\widetilde{v})+p^{-1}(\bar{\partial} \widetilde{v})-\rho\left(c^{1}\right)-\bar{\partial} \psi$ as in $(2.7)$, then we rewrite $\lim _{\varepsilon \rightarrow 0} \int_{\partial T_{\varepsilon}(D)} \nabla_{B S, v} \wedge$ $\pi^{*} \varphi$ as

$$
\lim _{\varepsilon \rightarrow 0} \int_{\partial T_{\varepsilon}(D)} \chi \gamma \pi^{*} \varphi+\lim _{\varepsilon \rightarrow 0} \int_{\partial T_{\varepsilon}(D)}(1-\chi) \gamma \pi^{*} \varphi+\operatorname{Res}_{s=0} \Phi(s) \varphi
$$

By Lemma 3.2 (ii) $\lim _{\varepsilon \rightarrow 0} \int_{\partial T_{\varepsilon}(D)} \chi \gamma \pi^{*} \varphi=I_{1}(p)$, where $I_{1}(p)$ is the right-hand side of Lemma 3.3. Next note that the zero order part of $\left(p^{0}(\widetilde{v})+p^{-1}(\bar{\partial} \widetilde{v})-\bar{\partial} \psi\right) d s$ is smooth in $X-U_{x_{0}}$ and $(1-\chi)\left(p^{0}(\widetilde{v})+p^{-1}(\bar{\partial} \widetilde{v})-\bar{\partial} \psi\right) d s$ is smooth in $U_{x_{0}}$. We conclude that $K_{\rho(c), \varphi}=\lim _{\varepsilon \rightarrow 0} \int_{\partial T_{\varepsilon}(D)}(1-\chi) \gamma \pi^{*} \varphi$ depends on $\rho\left(c^{1}\right)$ and $\chi$ but is independent of $p^{0}, p^{-1}$ and $\psi$. When $q \notin W_{1}$ and $\chi(q) \neq 0$ we must have $\rho\left(c^{1}\right)(q)=$ 0 since supp $\chi \subset W$ and $\rho\left(c^{1}\right) \equiv 0$ in $W$. Thus $K_{\rho(c), \varphi}$ does not depend on $\chi$. Now from (3.8),

$$
\lim _{\varepsilon \rightarrow 0} \int_{\partial T_{\varepsilon}(D)} \nabla_{B S, v} \wedge \pi^{*} \varphi=I_{1}(p)+K_{\rho(c), \varphi}+\underset{s=0}{\operatorname{Res}} \Phi(s) \varphi
$$

The left-hand side and $I_{1}(p)$ do not depend on $\rho(c)$, while $K_{\rho(c), \varphi}+\operatorname{Res}_{s=0} \Phi(s) \varphi$ depends only on $\rho(c)$ (and $\varphi$ ). Since $\rho(c)$ is an arbitrary action on $\lambda_{E}$ we must have $K_{\rho(c), \varphi}+\operatorname{Res}_{s=0} \Phi(s) \varphi=0$ and this proves Lemma 3.3.

\section{Some Consequences of the Curvature Formula}

Assuming the notations in the beginning of Sect. 3. Let $\theta_{B S}$ be the connection form of $\nabla_{B S}$ with respect to a nonzero holomorphic section $\sigma$ of $\lambda_{E}$ in $\Delta_{\varepsilon_{0}}$.

\section{Proposition 4.1.}

$$
\theta_{B S}=\frac{n r k(E)}{12} \frac{d s}{s}+\partial_{S}\left\{\psi_{0}+\psi_{1}|s|^{2} \log |s|\right\}
$$

where $\psi_{i}$ are $C^{\infty}$ functions on $\Delta_{\varepsilon_{0}}$.

Proof. The argument is similar to [BB, 12(c)]. By Theorem 3.1

$$
\overline{\partial_{S}}\left[\theta_{B S}-\frac{n r k(E)}{12} \frac{d s}{s}\right]=(-2 \pi i) \int_{X / S}\left[\operatorname{Td}\left(T Z, \nabla_{\omega^{-1}}\right) \operatorname{ch}\left(E, \nabla_{E}\right)\right]_{2} \text {. }
$$

By a partition of unity argument we can write on $X$

$$
(-2 \pi i)\left[\operatorname{Td}\left(T Z, \nabla_{\omega^{-1}}\right) \operatorname{ch}\left(E, \nabla_{E}\right)\right]_{2}=\bar{\partial} \partial \eta_{0}+\eta_{1},
$$

where $\eta_{l}$ are smooth on $X$ and $\eta_{1}$ vanishes in a neighborhood of the singular points $\Sigma$. Then $\int_{X / S} \eta_{1}$ is smooth on $\Delta_{\varepsilon_{0}}$, and hence by (4.1),

$$
\alpha=\theta_{B S}-\frac{n r k(E)}{12} \frac{d s}{s}-\partial_{S} \int_{X / S} \eta_{0}
$$


must be smooth on $\Delta_{\varepsilon_{0}}$ since $\overline{\partial_{S}}(\alpha)=\int_{X / S} \eta_{1}$. By [BB, Theorem 12.3]

$$
\int_{X / S} \eta_{0}=\gamma_{0}+\gamma_{1}|s|^{2} \log |s|
$$

where $\gamma_{0}, \gamma_{1}$ are smooth and this proves the proposition.

Note that $\partial_{S}\left\{\psi_{0}+\psi_{1}|s|^{2} \log |s|\right\}$ is continuous in $\Delta_{\varepsilon_{0}}$. Proposition 4.1 can be generalized to the case when $\operatorname{dim} S>1$ by using formulations analogous to [BB, Theorem 2.2].

Assume now $\nabla_{\omega}$ and $\nabla_{E}$ arise from hermitian metrics \|\|$_{\omega}$ and \|\|$_{E}$, so that $\nabla_{B S}$ coincides ([TTs]) with the corresponding $\nabla_{Q}$ associated to the Quillen metric \|\|$_{Q}$ on $\lambda_{E}$. In this case

$$
\theta_{B S}=\partial \log \|\sigma\|_{Q}
$$

and we conclude immediately from Proposition 4.1.

Corollary 4.2. $\left[B B\right.$, Theorem 2.2] There are $C^{\infty}$ functions $\psi_{i}$ on $\Delta_{\varepsilon_{0}}$ such that:

$$
\log \|\sigma\|_{Q}=\frac{n r k(E)}{12} \log |s|+\psi_{0}+\psi_{1}|s|^{2} \log |s|
$$

As remarked in the introduction, we have derived this estimate of the Quillen metric purely by local considerations. We compute next the curvature of a slightly different connection on $\lambda_{E}$.

In the neighborhoods $U$ of points of $\Sigma$ consider the metric on $T X$ which in local coordinates $\left(z_{0}, z_{1}\right)$ (cf. (1.1)) has the expression

$$
\left|d z_{0}\right|^{2}+\left|d z_{1}\right|^{2}
$$

By partition of 1 one then gets a metric \|\|$_{T X}$ on $X$ with the desired behavior at each $p_{i} \in \Sigma$. As in $[\mathrm{BB}, 6]$ denote the restriction of this metric to $\left.T Z\right|_{X-Z}$ by \|\|$_{T Z}$. Then

$$
\left\|\frac{\partial}{\partial z_{0}}-\frac{z_{1}}{z_{0}} \frac{\partial}{\partial z_{1}}\right\|_{T Z}^{2}=1+\left|\frac{z_{1}}{z_{0}}\right|^{2}
$$

and the curvature is

$$
R^{T Z}=\bar{\partial} \partial \log \left\|\frac{\partial}{\partial z_{0}}-\frac{z_{1}}{z_{0}} \frac{\partial}{\partial z_{1}}\right\|_{T Z}^{2}=\frac{\left(\bar{z}_{1} d \overline{z_{0}}-\overline{z_{0}} d \overline{z_{1}}\right)\left(z_{1} d z_{0}-z_{0} d z_{1}\right)}{|z|^{4}}
$$

In particular $R^{T Z} \wedge R^{T Z}=0$ in a neighborhood of the $p_{l}$ and so $T d\left(T Z,\|\|_{T Z}\right)$ exists as a locally $L^{1}$ current on $X$. Let $\nabla_{B S}^{1}$ be the Beilinson Schechtman connection on $\lambda_{E}$ associated to \|\|$_{T Z}$ and $\nabla_{E}$.

Proposition 4.3. $\nabla_{B S}^{1}$ is an $L^{1}$ connection on $\lambda_{E}$ and its curvature as a current is

$$
\frac{i}{2 \pi} \bar{\partial}_{S} \nabla_{B S}^{1}=\int_{X / S}\left[T d\left(T Z,\|\|_{T Z}\right) \operatorname{ch}\left(E, \nabla_{E}\right)\right]_{2}+\frac{n r k(E)}{6} \delta .
$$


Proof. Let \|\|$_{\omega}$ be a smooth metric on $\omega_{X / S}$ in $X$ such that in a neighborhood of each of the $p_{l} \in \Sigma$ and using the coordinates (1.1) we have

$$
\left\|\frac{d z_{0}}{z_{0}}\right\|_{\omega}^{2}=1
$$

\|\|$_{\omega}$ induces a metric on $T Z$ in $X-\Sigma$ denoted by \|\|$_{\omega^{-1}}$, and in particular

$$
\left\|\frac{\partial}{\partial z}\right\|_{\omega^{-1}}^{2}=\left|z_{0}\right|^{-2} \text {. }
$$

Let $\nabla_{B S}^{0}$ be the Beilinson Schechtman connection associated to \|\|$_{\omega^{-1}}$ and $\nabla_{E}$, then by Theorem 3.1,

$$
\frac{i}{2 \pi} \bar{\partial}_{S} \nabla_{B S}^{0}=\int_{X / S}\left[\mathrm{Td}\left(T Z,\|\|_{\omega^{-1}}\right) \operatorname{ch}\left(E, \nabla_{E}\right)\right]_{2}+\frac{n r k(E)}{12} \delta .
$$

Consider the family of metrics \|\|$_{t}=\mathrm{e}^{t \phi}\|\|_{\omega^{-1}}$ such that \|\|$_{1}=\|\|_{T Z}$. From (4.3) and (4.4) we have in a neighborhood of $p_{i}$,

$$
\phi=\log \frac{\|\|_{T Z}}{\|\|_{\omega^{-1}}}=\log \left(\left|z_{0}\right|^{2}+\left|z_{1}\right|^{2}\right) .
$$

Now by the Bott Chern variation formulas [TTs, (2.3)]

$$
\begin{aligned}
& \frac{i}{2 \pi}\left(\nabla_{B S}^{1}-\nabla_{B S}^{0}\right) \\
& \quad=\frac{i}{2 \pi} \int_{X / S}\left\{\frac{r k(E)}{6} \partial \phi \wedge c_{1}\left(\nabla_{\omega^{-1}}\right)+\frac{r k(E)}{12} \partial \phi \wedge \frac{i}{2 \pi} \bar{\partial} \partial \phi+\frac{1}{2} \partial \phi \wedge c_{1}\left(\nabla_{E}\right)\right\} .
\end{aligned}
$$

Using (4.6) and the fact that $c_{1}\left(\nabla_{\omega^{-1}}\right), c_{1}\left(\nabla_{E}\right)$ are smooth the integrand is clearly $L^{1}$ on $X$. Hence the fiber integral is $L^{1}$ on $S$ and we may calculate the $\overline{\partial_{S}}$ derivatives in the sense of currents. This is done in the same manner as in the proof of Theorem 3.1, and we obtain for a $C^{\infty}$ function $\psi$ with compact support on $S$.

$$
\begin{aligned}
\frac{i}{2 \pi} & {\left[\overline{\partial_{S}}\left(\nabla_{B S}^{1}-\nabla_{B S}^{0}\right)\right](\psi)=\frac{i}{2 \pi} \int_{S}\left(\nabla_{B S}^{1}-\nabla_{B S}^{0}\right)\left(\overline{\partial_{S}} \psi\right) } \\
& =\lim _{\varepsilon \rightarrow 0}-\frac{i}{2 \pi_{X}} \int_{-B_{\varepsilon}} \bar{\partial}\{\mu \wedge \psi\}+\lim _{\varepsilon \rightarrow 0} \frac{i}{2 \pi_{X}} \int_{-B_{\varepsilon}}(\bar{\partial} \mu) \wedge \psi,
\end{aligned}
$$

where $\mu$ is the integrand \{\} in (4.7) which only has singularities at $\Sigma$ and $B_{\varepsilon}$ denotes the $\varepsilon$ balls around each point of $\Sigma$. Hence we have

$$
\begin{aligned}
& n \lim _{\varepsilon \rightarrow 0} \frac{i}{2 \pi} \int_{\partial B_{\varepsilon}}\left\{\frac{r k(E)}{6} \partial \phi \wedge c_{1}\left(\nabla_{\omega^{-1}}\right)+\frac{r k(E)}{12} \partial \phi \wedge \frac{i}{2 \pi} \bar{\partial} \partial \phi+\frac{1}{2} \partial \phi \wedge c_{1}\left(\nabla_{E}\right)\right\} \psi \\
& \quad+\int_{S}\left\{\int_{X / S}\left[\operatorname{Td}\left(T_{Z},\|\|_{T Z}\right)-\operatorname{Td}\left(T_{Z},\|\|_{\omega_{-1}}\right)\right] \operatorname{ch}\left(E, \nabla_{E}\right)\right\} \psi
\end{aligned}
$$


where the last term follows from Proposition 2.2. In the limit term in (4.8) the only contributions with pole of order 3 is

$$
n \lim _{\varepsilon \rightarrow 0}\left(\frac{i}{2 \pi}\right)^{2} \frac{r k(E)}{12} \int_{\partial B_{\varepsilon}} \psi \partial \phi \wedge \bar{\partial} \partial \phi=n \lim _{\varepsilon \rightarrow 0} \frac{r k(E)}{12} \int_{\partial B_{\varepsilon}} \psi \omega^{0}=\frac{n r k(E)}{12} \psi(0) .
$$

Thus we obtain

$$
\begin{aligned}
& \frac{i}{2 \pi} \overline{\partial_{S}}\left(\nabla_{B S}^{1}-\nabla_{B S}^{0}\right) \\
& \quad=\int_{X / S}\left[\left\{\operatorname{Td}\left(T_{Z},\|\|_{T Z}\right)-\operatorname{Td}\left(T_{Z},\|\|_{\omega^{-1}}\right)\right\} \operatorname{ch}\left(E, \nabla_{E}\right)\right]_{2}+\frac{n r k(E)}{12} \delta .
\end{aligned}
$$

Proposition 4.1 now follows by adding (4.5) and (4.9).

Remark. It is also possible to calculate $\overline{\partial_{S}} \nabla_{B S}^{1}$ directly without making use of Theorem 3.1 and comparing with $\nabla_{B S}^{0}$. However the detailed calculations here are considerably more involved than that for Theorem 3.1 which is essentially contained in Lemma 2.3.

Let $c$ be a simple smooth curve in $\Delta_{\varepsilon_{0}}-\{0\}$ enclosing 0 once and let $\Delta$ be the interior of $c$. Let $\tau_{1}^{0}$ be the holonomy of the parallel transport of $\lambda_{E}$ for the connection $\nabla_{B S}^{1}$ once around $c$ in the positive sense.

Corollary 4.4. ([BB, Theorem 6.3])

$$
\tau_{1}^{0}=\exp \left\{2 \pi i\left(\iint_{\pi} T d\left(T Z,\|\|_{T Z}\right) \operatorname{ch}\left(E, \nabla_{E}\right)+\frac{n r k(E)}{6}\right)\right\} .
$$

Proof. Suppose $\lambda_{E}$ is trivialized in $\Delta_{\varepsilon_{0}}$ with local basis $e$ and suppose $f e$ is a parallel section:

$$
\nabla_{B S}^{1}(f e)=d f e+f \theta e=0
$$

where $\theta$ is the connection form with respect to $e$. Then over a portion of $c$ where $f \neq 0$,

$$
-\int_{t}^{t^{\prime}} \theta=-\int_{t}^{t^{\prime}} \frac{d f}{f}
$$

From this it follows readily that $\tau_{1}^{0}=e^{\int_{c}-\theta}$. Then by Proposition 4.3,

$$
\begin{aligned}
\tau_{1}^{0} & =\exp \left(-\int_{\Delta} \bar{\partial} \theta\right) \\
& =\exp \left\{2 \pi i\left(\iint_{\pi} \operatorname{Td}\left(T Z,\|\|_{T Z}\right) \operatorname{ch}\left(E, \nabla_{E}\right)+\frac{n r k(E)}{6}\right)\right\} .
\end{aligned}
$$

\section{References}

[BS] Beilinson, A., Schechtman, V.: Determinant bundles and Virasoro algebras. Commun. Math. Phys. 118, 651-701 (1988)

[BB] Bismut, J.M., Bost, J.B.: Fibrés déterminants, métriques de Quillen et dégénérescence des courbes. Acta. Math. 165 1-103 (1990) 
[D] Deligne, P.: Le déterminant de la cohomologie. Contemporary Mathematics, vol. 67, 93-178

[Mum] Mumford, D.: The red book of varieties and schemes. Lecture Notes in Math. V. 1358, Berlin, Heidelberg, New York: Springer 1988

[T] Tong, Yue Lin L.: Connections on determinant bundles. J. Alg. Geom., 443-486 (1993)

[TTs] Tong, Yue Lin L., Tsai, I-Hsun.: An identification of the connections of Quillen and Beilinson-Schechtman. Commun. Math. Phys. 159, 443-457 (1994)

Communicated by S.-T. Yau 